LAWRENCE LIVERMORE NAT IO N A L LABORATORY
Synthesis and Characterization of Mixed Metal Oxide Nanocomposite Energetic Materials

B.J. Clapsaddle, L. Zhao, A.E. Gash, J.H. Satcher Jr., K.J. Shea, M.L. Pantoya, R.L. Simpson

November 20, 2003

Materials Research society Fall Meeting 2003

Boston, MA, United States

November 30, 2003 through December 4, 2003 
This document was prepared as an account of work sponsored by an agency of the United States Government. Neither the United States Government nor the University of California nor any of their employees, makes any warranty, express or implied, or assumes any legal liability or responsibility for the accuracy, completeness, or usefulness of any information, apparatus, product, or process disclosed, or represents that its use would not infringe privately owned rights. Reference herein to any specific commercial product, process, or service by trade name, trademark, manufacturer, or otherwise, does not necessarily constitute or imply its endorsement, recommendation, or favoring by the United States Government or the University of California. The views and opinions of authors expressed herein do not necessarily state or reflect those of the United States Government or the University of California, and shall not be used for advertising or product endorsement purposes. 


\title{
Synthesis and Characterization of Mixed Metal Oxide Nanocomposite Energetic Materials
}

\author{
Brady J. Clapsaddle ${ }^{1}$, Lihua Zhao ${ }^{2}$, Alex E. Gash ${ }^{1}$, Joe H. Satcher Jr. ${ }^{1}$, Kenneth J. Shea ${ }^{2}$, \\ Michelle L. Pantoya ${ }^{3}$, and Randall L. Simpson ${ }^{1}$ \\ ${ }^{1}$ Lawrence Livermore National Laboratory, 7000 East Avenue, \\ Livermore, CA 94550, U.S.A. \\ ${ }^{2}$ Department of Chemistry, University of California, Irvine, Frederick Reines Hall \\ Irvine, California, 92612 \\ ${ }^{3}$ Department of Mechanical Engineering, Texas Tech University, Box 41021, \\ Lubbock, Texas 79409-1021, U.S.A.
}

\begin{abstract}
In the field of composite energetic materials, properties such as ingredient distribution, particle size, and morphology, affect both sensitivity and performance. Since the reaction kinetics of composite energetic materials are typically controlled by the mass transport rates between reactants, one would anticipate new and potentially exceptional performance from energetic nanocomposites. We have developed a new method of making nanostructured energetic materials, specifically explosives, propellants, and pyrotechnics, using sol-gel chemistry. A novel sol-gel approach has proven successful in preparing metal oxide/silicon oxide nanocomposites in which the metal oxide is the major component. Two of the metal oxides are tungsten trioxide and iron(III) oxide, both of which are of interest in the field of energetic materials. Furthermore, due to the large availability of organically functionalized silanes, the silicon oxide phase can be used as a unique way of introducing organic additives into the bulk metal oxide materials. As a result, the desired organic functionality is well dispersed throughout the composite material on the nanoscale. By introducing a fuel metal into the metal oxide/silicon oxide matrix, energetic materials based on thermite reactions can be fabricated. The resulting nanoscale distribution of all the ingredients displays energetic properties not seen in its microscale counterparts due to the expected increase of mass transport rates between the reactants. The synthesis and characterization of these metal oxide/silicon oxide nanocomposites and their performance as energetic materials will be discussed.
\end{abstract}

\section{INTRODUCTION}

Since the invention of black powder, one thousand years ago, the technology for making solid energetic materials has remained either the physical mixing of solid oxidizers and fuels (e.g. black powder), or the incorporation of oxidizing and fuel moieties into one molecule (e.g., trinitrotoluene (TNT)). The basic distinctions between these energetic composites and energetic materials made from monomolecular approaches are as follows. In composite systems, desired energy properties can be attained through readily varied ratios of oxidizer and fuels. A complete balance between the oxidizer and fuel may be reached to maximize energy density. However, due to the granular nature of composite energetic materials, reaction kinetics are typically controlled by the mass transport rates between reactants. Hence, although composites may have extreme energy densities, the release rate of that energy is below that which may be attained in a chemical kinetics controlled process. In monomolecular energetic materials the rate of energy release is primarily controlled by chemical kinetics, and not by mass transport. Therefore, 
monomolecular materials can have much greater power than composite energetic materials. A major limitation with these materials is the total energy density achievable. Therefore, it is desirable to combine the excellent thermodynamics of composite energetic materials with the rapid kinetics of the monomolecular energetic materials. One possible way to do this is to mix the components of composite energetic materials on a size scale which will limit the effects of mass transport on the reactants, thus providing kinetics similar to those obtained in monomolecular energetic materials.

We have previously prepared pyrotechnic and explosive composites based on thermite reactions whose fuel and oxidizer constituents are intimately mixed on the nanometer-sized scale [1-2]. These nanoenergetic composites, in an attempt to prepare a high energy, high power nanoenergetic composite as discussed above, are prepared by taking advantage of the unique nano-architechture and mixing properties provided by sol-gel chemistry. One limitation inherent in any thermite energetic, however, is the inability of the energetic material to do pressure/ volume-work on an object. Thermites release energy in the form of heat and light, but are unable to move objects. Typically, work can be done by a rapidly produced gas that is released during the energetic reaction. In the case of the thermite energetic composite, a "gas generator" can be added to the composite mixture, however, this additive will be subject to the same mass transport issues mentioned above.

This paper will discuss the preparation of energetic nanocomposites containing organically modified iron(III) oxide-silica oxidizing phases. All nanocomposites have been synthesized using sol-gel chemistry in which aluminum metal particles are intimately mixed with the iron(III) oxide-silicon oxide phase. The incorporation of organic functionality through the silicon oxide precursors results in the intimate mixing of a gas generator into the energetic composite on the nanoscale. The release of gas upon ignition of the nanocomposite material is observed.

\section{EXPERIMENTAL DETAILS}

\section{Synthesis of organic functionalized mixed metal oxide energetic nanocomposites}

In a typical reaction, $\mathrm{FeCl}_{3} \cdot 6 \mathrm{H}_{2} \mathrm{O}(0.84 \mathrm{~g}, 3 \mathrm{mmol})$ was dissolved with stirring in $3.8 \mathrm{~g}$ of 200 proof ethanol in a polyethylene vial to give a yellow-brown solution. Once the $\mathrm{FeCl}_{3} \cdot 6 \mathrm{H}_{2} \mathrm{O}$ was dissolved, the desired amount of organosilane (Fe/Si atomic molar ratio of 3-5) was added to the solution and the resulting mixture allowed to stir for 10 minutes. To induce gelation, a proton scavenging organic epoxide, propylene oxide (PO; $28 \mathrm{mmol})$, was then added to the sol in at least two separate aliquots. (CAUTION: addition of $\mathrm{PO}$ to a $\mathrm{FeCl}_{3} \cdot 6 \mathrm{H}_{2} \mathrm{O}$ solution is accompanied by significant heat generation, which in some cases leads to flash boiling of the synthesis solution and should only be used in a well ventilated lab space. The authors recommend the careful addition of $\mathrm{PO}$ to the $\mathrm{FeCl}_{3} \cdot 6 \mathrm{H}_{2} \mathrm{O}$ /organosilane solution in two or three aliquots, allowing for sufficient cooling time between additions.) Before the addition of the last aliquot of epoxide, $2 \mu \mathrm{m}$ aluminum metal $(6 \mathrm{mmol} ; \mathrm{Al} / \mathrm{Fe}=0.5)$ was added to the sol and the mixture stirred for 15 minutes to ensure thorough mixing. While continuing to gently stir, the final aliquot of PO was added and the sol allowed to gel.

\section{Processing of organic/inorganic hybrid gels}


All gels remained covered and were aged for at least 48 hours after the initial gelation. Following aging, each gel was subjected to a pore-washing/solvent exchange step in 200-proof ethanol for $3-5$ days. During this time, the wash solution was replaced at least three times with fresh ethanol. For aerogel preparation, the solvent-exchanged gels were processed in a Polaron ${ }^{\mathrm{TM}}$ supercritical point dryer. The ethanol in the wet-gel pores was exchanged for $\mathrm{CO}_{2}(l)$ for 3 - 4 days at $\sim 12{ }^{\circ} \mathrm{C}$, after which the temperature of the vessel was ramped to $\sim 45^{\circ} \mathrm{C}$ while maintaining a pressure of $\sim 100$ bar. The vessel was then depressurized at a rate of about 7 $\mathrm{bar} / \mathrm{hr}$. For xerogel preparation, the wet gels were solvent exchanged with pentane and then allowed to dry ambiently for 2-3 days. The resulting xerogel monoliths were then placed in a vacuum oven at $85^{\circ} \mathrm{C}$ for 24 hours to complete the drying process.

\section{Physical characterization of nanocomposites}

Infrared spectroscopy was performed with $\mathrm{KBr}$ pellets on a ThermoNicolet Nexus 4000 FTIR spectrometer under a nitrogen purge. All spectra were collected in transmission mode at 4 $\mathrm{cm}^{-1}$ resolution for 128 scans.

Surface area determination, pore volume, and pore size analysis were performed by BET (Brunauer-Emmett-Teller) and BJH (Barrett-Joyner-Halenda) methods using an ASAP 2000 surface area analyzer (Micromeritics Instrument Corp.). Samples of approximately 0.1-0.2 g were heated to $100{ }^{\circ} \mathrm{C}$ under vacuum (10-5 torr) for at least $24 \mathrm{~h}$ to remove all adsorbed species. Nitrogen adsorption data were taken at five relative pressures from 0.05 to 0.20 at $77 \mathrm{~K}$, to calculate the surface area by BET theory.

Transmission electron microscopy (TEM) was performed on a Philips CM300FEG operating at $300 \mathrm{keV}$ using zero loss energy filtering with a Gatan energy imaging filter (GIF) to remove inelastic scattering. The images were taken under bright field conditions and slightly defocused to increase contrast. The images were also recorded on a $2 \mathrm{~K} \times 2 \mathrm{~K}$ CCD camera attached to the GIF. Energy filtered TEM (EFTEM) element maps were obtained by electron energy loss spectroscopy (EELS) in tandem with the Philips CM300FEG TEM microscope. All EELS measurements were made with a Gatan model 607 electron energy-loss spectrometer attached to the microscope and were made at the $\mathrm{Si}-\mathrm{L}_{2,3}$ and F-K edges.

Scanning electron microscopy (SEM) was carried out using a Hitachi S-4500 cold field emission microscope. Typical accelerating voltages used ranged from 1.8 to $6 \mathrm{kV}$ and depended on sample conductivity.

\section{DISCUSSION}

\section{Synthesis and characterization of energetic nanocomposites}

We have previously reported a variety of transition metal oxide gels prepared by adding an organic epoxide to a variety of metal salt solutions, including $\mathrm{FeCl}_{3} \cdot 6 \mathrm{H}_{2} \mathrm{O}$ [3-5]. The epoxide acts as a gelation promoter by scavenging protons from solution through a ring-opening reaction

with the nucleophilic anion of the $\mathrm{Fe}^{3+}$ salt, the mechanism of which has been discussed in detail elsewhere [3]. We have recently used this epoxide addition method for the synthesis of Fe-Si mixed oxide composites. The addition of an organic epoxide to an ethanolic solution of $\mathrm{FeCl}_{3} \cdot 6 \mathrm{H}_{2} \mathrm{O}$ and either TMOS or TEOS resulted in the first example of Fe-Si mixed oxide nanocomposites in which iron(III) oxide is the major component [6]. 


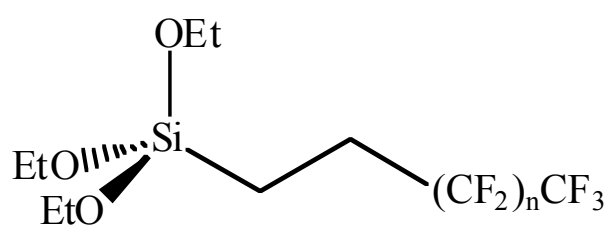

TDFTES $(n=5)$

HDFTES $(n=7)$

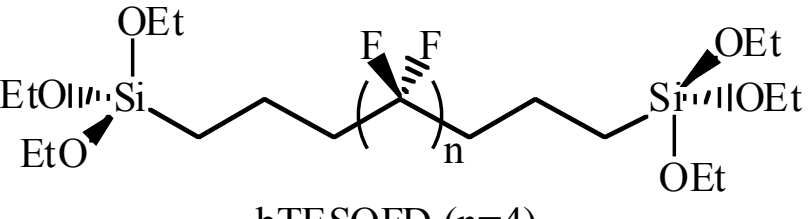

bTESOFD $(n=4)$

bTESHFT $(n=8)$

This technique has now been expanded to the synthesis of organic/inorganic hybrid composites by using a variety of organically functionalized silsesquioxanes and bissilsesquioxanes, shown above. The resulting materials are organic/inorganic hybrid composites in which all the components are well dispersed throughout the bulk material. The organic functionality chosen for these materials were comprised of a variety of fluoroalkanes due to the known energetic reaction between aluminum metal and $\mathrm{CF}_{2}$ moieties. Two silsesquioxanes used in these syntheses were (tridecafluoro-1,1,2,2-tetrahydrooctyl)triethoxysilane (TDFTES) and (heptadecafluoro-1,1,2,2-tetrahydrodectyl) triethoxysilane (HDFTES). The fluorinated bissiloxanes, 1,10-bis(triethoxysilyl)octafluorodecane (bTESOFD) and 1,14-bis(triethoxysilyl)-hexadecafluorotetradecane (bTESHFT) have also been synthesized and incorporated into iron(III) oxide composite materials [7].

The degree of dispersion between the phases throughout the bulk material is of interest in any composite material, but is especially important for the nanostructured energetic materials. The nanostructure of the organic functionalized metal oxide materials was characterized by TEM and EFTEM to determine the degree of dispersion of the organosilica phase in the material. Figure 1 contains images of a $\mathrm{Fe} / \mathrm{Si}=5$ aerogel made with the silica precursor HDFTES. The material is a collection of nanoparticles interconnected to form a mesoporous structure. The size of the particles appears to be fairly uniform throughout the gel, with particles sizes ranging from $2-5 \mathrm{~nm}$ in diameter. No evidence of phase separation between Fe- and Si-oxides is apparent from TEM analysis or EFTEM analysis. As a result, dispersion of the organo-silica phase appears to be uniform throughout the material on the nanoscale, as can be seen from the fluorine and silicon element maps in Figure 1. In fact comparison of individual particles between the TEM bright field image and EFTEM images shows there to be a mix of both organo-silica and iron(III) oxide in a significant number of particles. Incorporation of aluminum metal into the organically functionalized oxide matrix is accomplished through gelation of the matrix around the aluminum particles. The aluminum used in these studies was comprised of $2 \mu \mathrm{m}$ aluminum particles introduced into the sol just prior to gelation. Figure 2 shows an SEM of the final energetic composite. The $\mathrm{Al}$ metal particles are completely coated by the oxidizing matrix, thus showing the degree of mixing achievable for these energetic composite materials.

\section{Phenomenological burn properties}

Phenomenological burn observations indicate that the organic functionalized $\mathrm{Fe} / \mathrm{Si}$ mixed oxide nanocomposites burn very rapidly and violently, essentially to completion, with the generation of significant amounts of gas. Figure 3 shows a comparison of the ignition of an energetic nanocomposite with and without organic functionalization. The still image of the energetic nanocomposite without organic functionalization exhibits rapid ignition and emission 

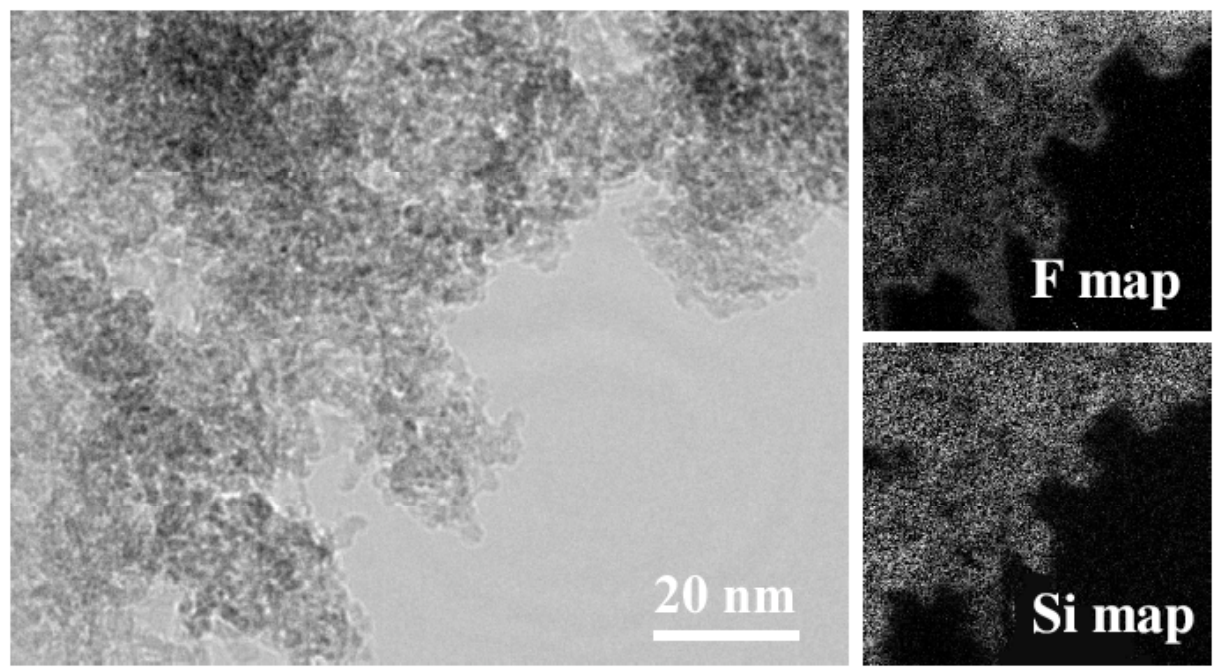

Figure 1. TEM micrograph of an $\mathrm{Fe} / \mathrm{Si}=5$ organic/inorganic aerogel composite. Fluorine and silicon element maps show the dispersion of the organosilica phase throughout the material.

of light and heat. The still image of the energetic nanocomposite with organic functionalization also exhibits these characteristics, but it also exhibits hot particle ejection due to the production of gas upon ignition. This reaction is very exothermic and results in the production of very high temperatures, intense light, and pressure from the generation of the gaseous byproducts from the decomposition of the organic moieties. Future work will focus on characterization of the products and measurement of burn rates, temperatures, and pressure generated by such materials.

\section{CONCLUSIONS}

We have successfully synthesized energetic nanocomposites using sol-gel methodology that

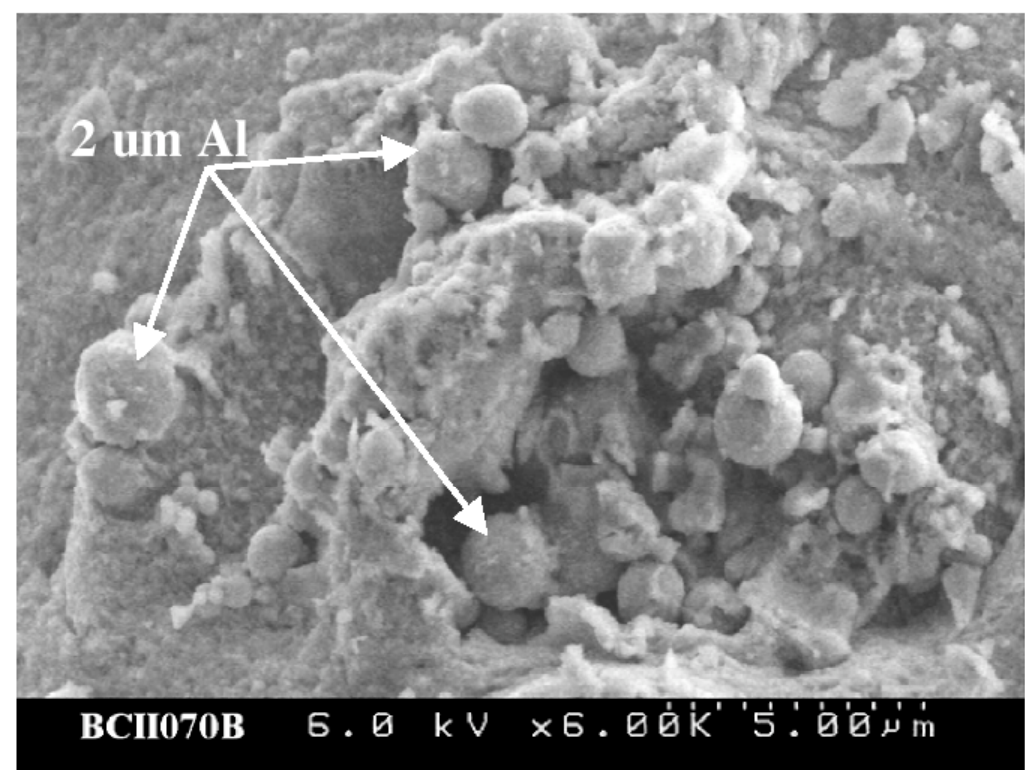

Figure 2. $\mathrm{SEM}$ images of an $\mathrm{Fe} / \mathrm{Si}=5$ organic/inorganic energetic nanocomposite with $2 \mu \mathrm{m} \mathrm{Al}$ metal particles. 

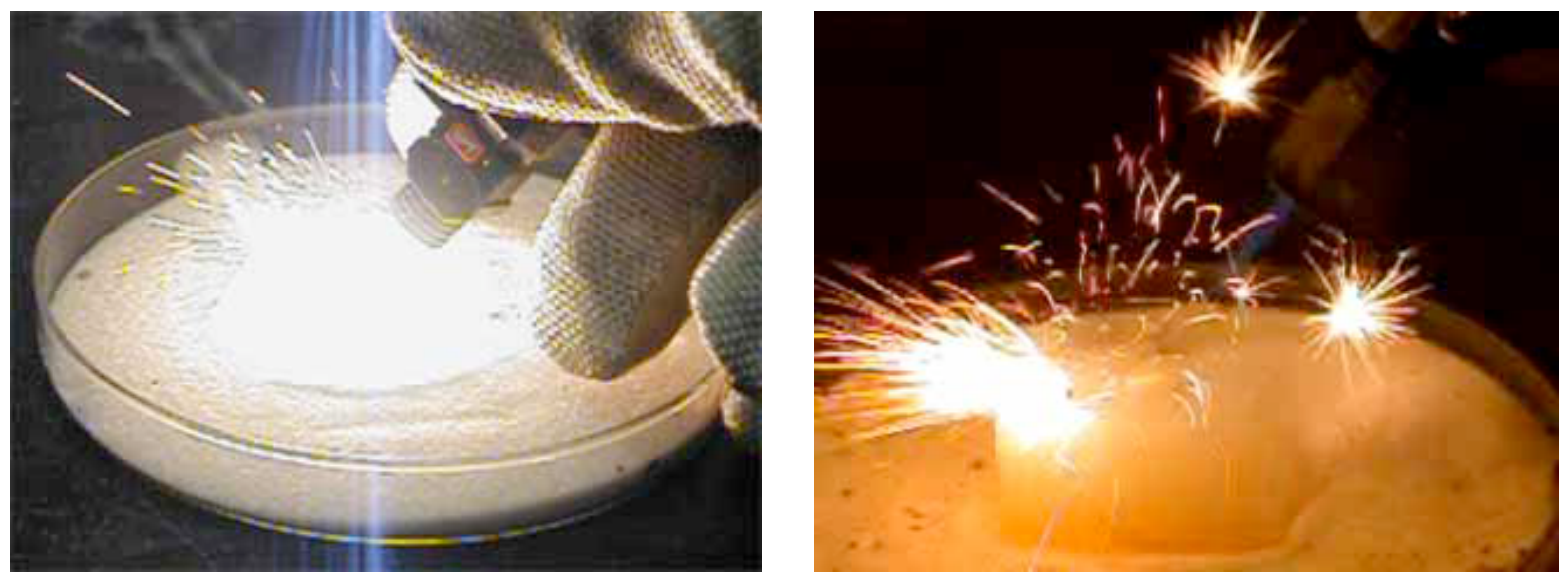

Figure 3. Still images of thermal ignition of a $25 \mathrm{mg}$ samples of iron(III) oxide/2 $\mu \mathrm{m} \mathrm{Al}$ (left) and $\mathrm{Fe} / \mathrm{Si}=5$ organo/2 $\mu \mathrm{m} \mathrm{Al}$ (right) energetic nanocomposites.

incorporate gas generators through an organic functionalized iron(III) oxide-silica oxidizing phase. The energetic materials display and intimate mixing on the nano-scale of all components. The material is exothermic when ignited, displaying hot particle ejection caused by the rapid generation of gas from decomposition of the organic functionality. These energetic nanocomposites have potential applications as pyrotechnics and propellants.

\section{ACKNOWLEDGEMENTS}

This Work was performed under the auspices of the U.S. Department of Energy by the University of California Lawrence Livermore National Laboratory under contract No. W-7405Eng-48. The authors would like to thank Jennifer S. Harper for TEM images and Jim Ferreira for SEM images.

\section{REFERENCES}

1. T. M. Tillotson, A. E. Gash, R. L. Simpson, L. W. Hrubesh, J. H. Satcher Jr., J. F. Poco, J.Non-Cryst. Solids 285, 338 (2001).

2. A. E. Gash, R. L. Simpson, J. H. Satcher Jr., Proceedings of the $27^{\text {th }}$ International Pyrotechnics Seminar, July 15-21, 2001, Grand Junction, CO, U.S.A.

3. A. E. Gash, T. M. Tillotson, J. H. Satcher Jr., J. F. Poco, L. W. Hrubesh, R. L. Simpson, Chem. Mater. 13, 999 (2001).

4. A. E. Gash, T. M. Tillotson, J. H. Satcher Jr., L. W. Hrubesh, R. L. Simpson, J. Non-Cryst. Solids 285, 22 (2001).

5. A. E. Gash, J. H. Satcher Jr., R. L. Simpson, Chem. Mater. 15, 3268 (2003).

6. B. J. Clapsaddle, A. E. Gash, J. H. Satcher, R. L. Simpson, J. Non-Cryst. Solids 331, 190 (2003).

7. L. Zhao, K. J. Shea, B. J. Clapsaddle, A. E. Gash, J. H. Satcher, R. L. Simpson, manuscript in preparation. 